\title{
Clinicopathological Study of Malignant Lymphomas
}

\author{
Dr Impana B.D ${ }^{1}$, Dr Teerthanath $\mathrm{S}^{2}$, Dr Sunil Kumar $\mathrm{Y}^{3}$, \\ Dr Kishan Prasad H.L ${ }^{4}$, Dr Jayaprakash Shetty $\mathrm{K}^{5}$ \\ Assistant Professor ${ }^{1}$, Professor ${ }^{2}$, Assosciate Professor ${ }^{3}$, Assosciate Professor ${ }^{4}$, Professor And $\mathrm{Head}^{5}$ \\ ${ }^{1}$ Department OfPathology, KVG Medical College And Hospital, \\ ${ }^{2,3,4,5}$ Department $O f$ Pathology, K S Hegde Medical Academy
}

\begin{abstract}
Background: Lymphoma is the most common form of haematological malignancy in the developed and developing countries. There is a remarkable increase in the incidence of lymphomas worldwide. Lymphomas are divided as Hodgkin lymphoma (HL) andnon-Hodgkin lymphoma (NHL).

Aims and objectives of the study:To determine the incidence and to study the clinical and pathological patterns of lymphoma (Hodgkin and non-Hodgkin lymphoma).

Materials and methods: A total of 50 cases were studied from June 2013 to June 2015 prospectively at a tertiary health care centre, Mangalore. Patient's data were retrieved from hospital records. The histopathological examination of lymph node and/or other involved organ was done and diagnosis was made. Immunohistochemical analysis was done for all the cases.

Statistical analysis: Chi-square test was used to find association between the different subtypes and SPSS version 16 was used for statistical analysis.

Results: The study group consisted of 50 cases of malignant lymphomas. Among all 50 cases of lymphomas, NHL comprised of $84 \%$, while HL comprised of 16\%. The age distribution of the patients ranged from 9 to 76 years with a mean age of 51.3 years. Both NHL and HL had a male preponderance with $66.7 \%$ and $62.5 \%$ respectively. The most common symptoms encountered in the study was lymph node enlargement in 64\%. Both nodal and extranodal lymphomas accounted for $50 \%$ of cases each.

Among NHL, 59.5\% had extranodal involvement and 40.5\% had nodal involvement. All the cases of HL were of nodal type. Most common site of extranodal lymphoma was gastrointestinal tract (24\%). The most common group of lymph nodes involved was cervical group followed by axillary group in both HL and NHL. In NHL, Bcell type was $71.4 \%$ and T-cell type was $28.6 \%$. Among B-cell lymphoma, diffuse large B cell lymphoma (DLBCL) was the most common histological subtype (66.7\%). Among T-cell, extranodal NK/T cell lymphoma was most common (25.1\%) type. MCCHL (50\%) was the most common in HL.

Conclusion: Lymphomas are potentially curable malignancies with the help of proper subtyping based on morphology and immunohistochemical diagnosis.
\end{abstract}

Keywords:Hodgkin lymphoma, non-Hodgkin lymphoma, Diffuse large B cell lymphoma

\section{Introduction}

Lymphomas are defined as malignant disorder of cells residing in lymphoid tissue (B cell, T cell, NK cell). Lymphoid-haemopoietic malignancies are a significant category of neoplasms in India representing $9.5 \%$ of all cancers in men and $5.5 \%$ in women. ${ }^{1}$ They are divided into Hodgkin lymphoma (HL) and non-Hodgkin lymphoma (NHL). Lymphomas can occur at any age. HL has a bimodal presentation with one peak in early years of life and other after middle age. Patients with lymphoma usually present with constitutional symptoms of weight loss, fever and night sweats or with enlarged lymph nodes and its pressure effects. Symptoms may also develop due to involvement of extranodal sites such as gastrointestinal tract, central nervous system, skin, liver, or bone, thus leading to atypical presentations. ${ }^{2}$ In Asian population, NHL is the most common form of lymphoid neoplasm than HL. NHL is the 8th most commonly diagnosed cancer in men and 11 th in women. ${ }^{3}$ The relative frequencies of the various subtypes of NHL vary across geographical regions and the pattern in India show significant differences from the rest of the world. T cell lymphomas are more common in south India as compared to the west. ${ }^{4}$

\section{Materials And Methods}

The present study deals with clinicopathological study of malignant lymphomas. A total of 50 cases were studied from June 2013 to June 2015 in a tertiary care centre, Mangalore.Patient's data regarding clinical history, examination, investigations and diagnosis were retrieved from hospital records. Patients presenting with symptoms of lymphadenopathy, and other systemic organ involvement was examined and biopsy of the affected lymph node or the organ was taken by the surgeons, after receiving an informed consent from the patient by 
their local language. The histopathological examination of lymph node and/or other involved organ was done and diagnosis was made.

Procedure: Biopsy and excision specimens were fixed in formalin (10\% solution of formaldehyde in buffered alcohol) and then processed. Section of 4 micrometer were mounted on glass slide and stained with hematoxylin and eosin $(\mathrm{H} \& \mathrm{E})$ stain. Special stains was done wherever necessary. Immunohistochemistry was done in all cases for further typing and classification. All the biopsy specimens of enlarged lymph node and suspicious extranodal sites of lymphomas, which were sent to the department of Pathology were included in the study. Patients unwilling to give consent were excluded.

\section{Results}

The study group consisted of 50 cases of malignant lymphomas. NHL comprised of 84\%(42 cases), while HL comprised of $16 \%$ (8 cases). (Fig 1)

Fig 1:Pie chart showing distribution of lymphomas

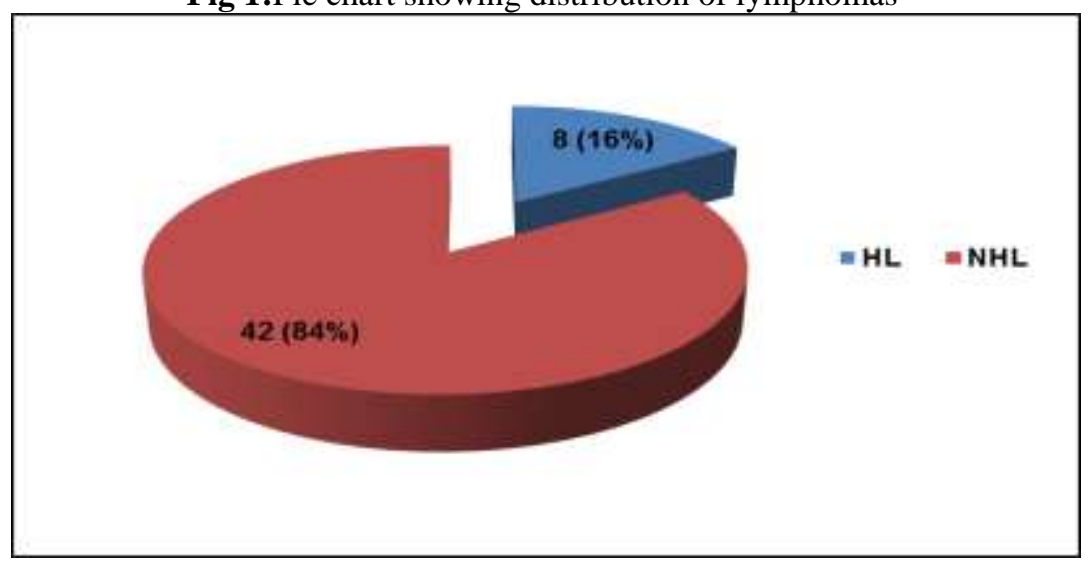

The age range in NHL was between 9 to 76 years with a mean age of 52.5 years. The age range of HL was between 13 to 60 years with a mean age of 44.7 years. Majority of the patients were males ( 33 cases), with a male to female ratio of 1.9:1. The male to female ratio in NHL was 2:1, and in HL was 1.7:1.

The most common symptoms encountered in the study was lymph node enlargement in $64 \%$ and B symptoms in $48 \%$. Hepatomegaly was seen in $34 \%$ and splenomegaly in $30 \%$ of cases. Other symptoms were abdominal pain $(30 \%)$, nasal mass $(8 \%)$, skin related $(8 \%)$ and CNS related symptoms $(4 \%)$.

Both nodal and extranodal lymphomas accounted for $50 \%$ of cases each. Among NHL, 59.5\% had extranodal involvement and $40.5 \%$ had nodal involvement. All the cases of HL were of nodal type. Most common site of extranodal lymphoma was gastrointestinal tract $(24 \%)$, followed by bone (20\%), nose $(16 \%)$, skin $(16 \%)$, CNS $(8 \%)$, thyroid $(8 \%)$ and tonsil $(8 \%)$.

The most common group of lymph nodes involved was cervical group followed by axillary group in both HL and NHL. In NHL, pan-B cell markers were positive in 30 cases (71.4\%) and pan-T cell markers were positive in 12 cases $(28.6 \%)$. (Fig 2)

Fig 2: Pie chart showing incidence of B and T-cell lymphoma in NHL

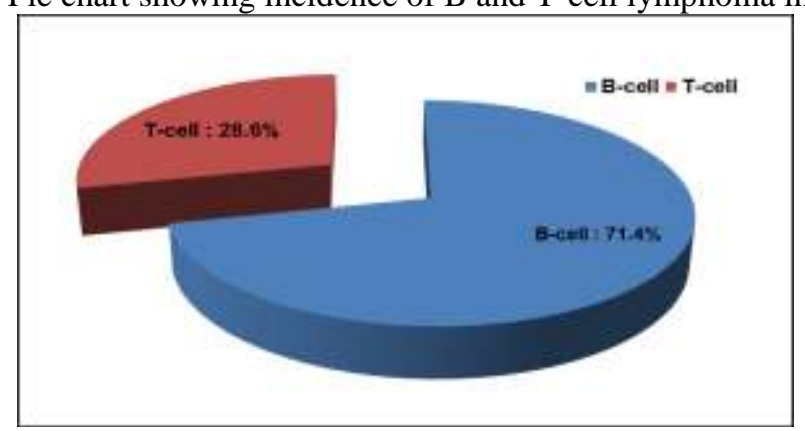

Among B cell type of NHL, DLBCL was the most common histological subtype accounting $66.7 \%$ followed by $10 \%$ of FL. (Fig 3 ) 
Fig 3:Histological subtypes of B-cell NHL

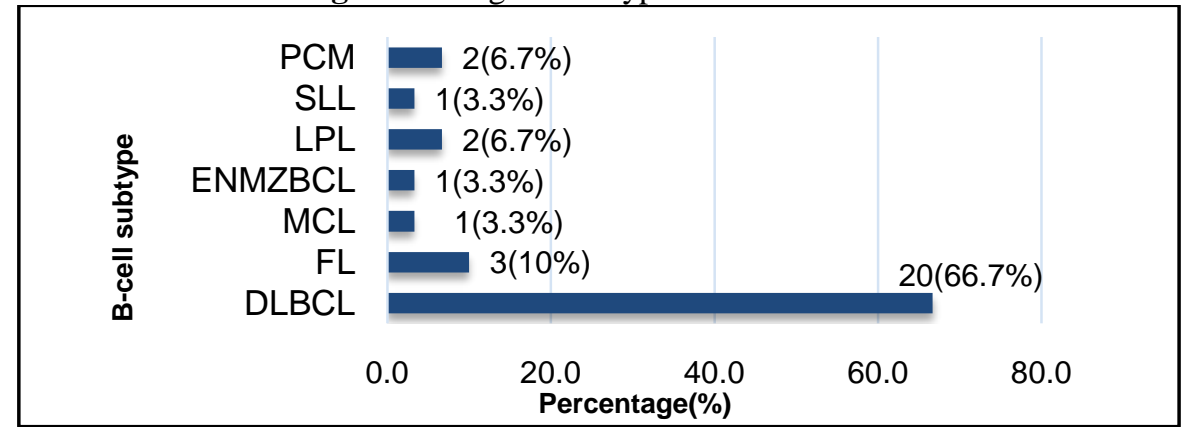

Among T-cell type of NHL, Extranodal NK/T cell lymphoma was the most common histological type comprising of $25.1 \%$, followed by anaplastic large T-cell lymphoma (16.7\%) and mycosis fungoides $(16.7 \%)$ (Fig 4).

Fig 4:Histological subtypes of T-cell NHL

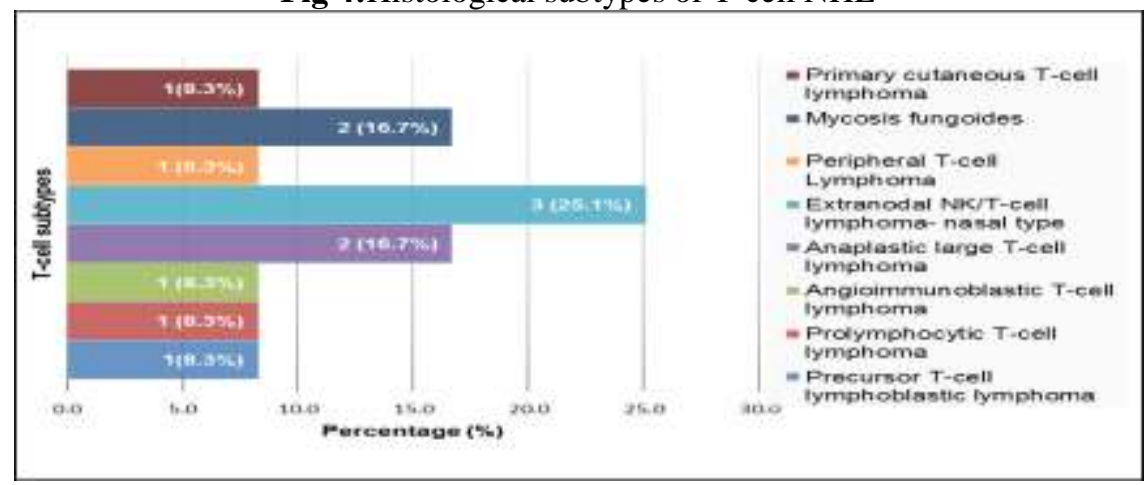

According to International working formulation classification, majority of the NHL were of intermediate grade $(59.5 \%)$, followed by low grade (19\%) and high grade $(11.9 \%)$. (Table 1$)$

Table 1:Grading of NHL based on International Working Formulation Classification

\begin{tabular}{|l|c|c|c|}
\hline Grade of NHL & Cases n=42 & $\begin{array}{c}\text { Cases reactive to Pan B } \\
\text { markers n=30 }\end{array}$ & $\begin{array}{c}\text { Cases reactive to Pan } \\
\text { T markers n=12 }\end{array}$ \\
\hline Low grade & $8(19 \%)$ & $7(23.3 \%)$ & $1(8.3 \%)$ \\
\hline Intermediate grade & $25(59.5 \%)$ & $19(63.3 \%)$ & $6(50 \%)$ \\
\hline High grade & $5(11.9 \%)$ & $2(6.7 \%)$ & $3(25 \%)$ \\
\hline Miscellaneous & $4(9.5 \%)$ & $2(6.7 \%)$ & $2(16.7 \%)$ \\
\hline
\end{tabular}

In HL, MCCHL (50\%) was the most common type encountered followed by nodular sclerosis $(37.5 \%)$ and lymphocyte rich (37.5\%). (Fig 5)

Fig 5:Pie chart showing histological subtypes of HL

$(37.5 \%)$

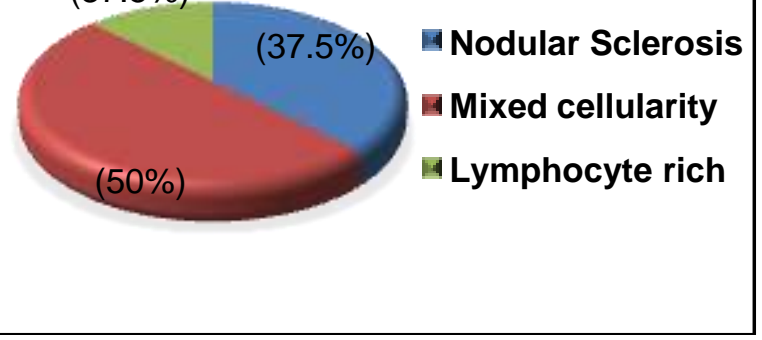


Childhood lymphomas was encountered in 2 cases, of which one case was precursor T-cell lymphoblastic lymphoma and the other was MCCHL.

Bone marrow involvement was seen in $22 \%$ of lymphomas. Among which 10 cases (23.8\%) was NHL and 1 case $(12.5 \%)$ was HL.Clinical staging was done using Ann-Arbor for both NHL and HL. Among 50 cases of lymphomas, $52 \%$ were in stage IV, followed by $26 \%$ in stage III, $16 \%$ in stage II and $6 \%$ in stage I.

\section{Discussion}

In the present study, 50 cases of malignant lymphomas were analysed. NHL was the most common type of lymphoma accounting for $84 \%$ of cases and HL was seen in $16 \%$ of cases. This is in concordance with various studies conducted by Mozaffer et al $(2008)^{2}$, Yang et al (2011) $)^{5}$, and Waravita et al (2015) ${ }^{6}$. However there was a mild increase in HL than NHL in the study conducted by Claude et al $(2014)^{7}$ which was $24 \%$ and $76 \%$ respectively.

In our study, the age range in NHL was from 9 to 76 years with a mean age of 52.5 years and the age range of HL was 13 to 60 years with a mean age of 44.7 years.

The mean age in NHL was comparable with Wei-Liang et al $(2010)^{8}$ and Waravita et al $(2015)^{6}$, whereas it was lower in the study by Yang et al $(2011)^{5}$ which was 44.1 years.

The mean age of HL was comparable with Waravita et al $(2015)^{6}$, however lower mean age was encountered in study conducted by Yang et al $(2011)^{5}$ and Wei-Liang et al $(2010)^{8}$.

NHL was more commonly seen in elderly population predominantly than in younger adults. Thus, age presents to be a strong risk factor in NHL. ${ }^{3}$

A single age peak of HL was seen in the elderly $\left(6^{\text {th }}\right.$ decade) population in our study which was also encountered by Aoki et al in Japan. ${ }^{9}$

In the study group, malignant lymphomas were common in men (66\%) than in women (34\%) with a male to female ratio of 1.9:1. Both NHL and HL showed a male preponderance which was in agreement with other studies by Wei-Liang et al (2010) ${ }^{8}$, Yang et al $(2011)^{5}$ and Waravita et al(2015) . $^{6}$

ML can present with nodal or extranodal manifestations. Majority of the cases of NHL showed extranodal involvement $(59.5 \%)$, whereas all the cases of HL were of nodal type. This was similar to studies by Jin man et al (2011) ${ }^{10}$ and Sun et al (2012) ${ }^{11}$, who also observed higher incidence of extranodal lymphomas among NHL. However Santosh et al(2013) ${ }^{12}$ found a higher incidence of nodal type of NHL.

According to $\mathrm{Naz}$ et al, it is well established that the extranodal lymphomas have a higher incidence in Asian population, as seen in our study. ${ }^{13}$

The frequency of extranodal NHL varies in different parts of the world. Studies from Western countries have reported the occurrence of extranodal NHL as $24-48 \%$ of all NHL. However, this incidence is higher in Asia, for example, Pakistan (42\%), Kuwait (45\%), Japan (46.6\%), Korea (55\%), Thailand (58.7\%), and China $(44.9 \%-61.4 \%)$. The fluctuating frequency of extranodal lymphomas may be caused by genetic and ethnic factors. ${ }^{5}$

In nodal lymphomas of NHL, cervical group of lymph nodes were most commonly involved, followed by axillary, inguinal and abdominal group of lymph nodes. This was in concordance with studies by Santosh et al $(2013)^{12}$ and Sudipta et al $(2010)^{1}$.

In HL, the most common lymph node involved was cervical group followed by axillary and inguinal group of lymph nodes. Cervical groups of lymph node was most commonly encountered in the study done by Sudipta et al (2010). ${ }^{1}$

The frequency of extranodal NHL varies in different parts of the world. Gastrointestinal tract was the most common site and was involved in $24 \%$ of all extranodal NHL. This was followed by nose (16.0\%), bone $(20.0 \%)$, skin $(16.0 \%)$, CNS $(8.0 \%)$ tonsil $(8.0 \%)$ and thyroid $(8.0 \%)$. Other studies like Yaqo et al $(2011)^{14}$, Jin Man et al $(2011)^{10}$, Sun et al $(2012)^{11}$ and Santosh et al (2013) ${ }^{12}$ also encountered gastrointestinal tract as the most common site of NHL. The rationale for this may be the role of environmental factors in H. pylori infection. One of the case in our study also showed positivity for H. pylori. Factors such as overcrowding, hygiene, water supply and sanitation have been linked with H. pylori association of gastric lymphomas. ${ }^{8}$

The most common type of lymphoma in extranodal site was DLBCL which was also encountered by Yang et al $(2011)^{5}$ and Waravita et al $(2015)^{6}$. According to Yaqo et al $(2011)^{14}$, the higher incidence of DLBCL can be attributed to possible large cell transformation of prior undiagnosed MALT lymphomas.

Hepatomegaly and splenomegaly are commonly encountered in higher stage of lymphomas. The incidence of hepatomegaly in the present study was $40.5 \%$ in NHL and $37.5 \%$ in HL. Splenomegaly was seen in $35.7 \%$ of NHL and $12.5 \%$ of HL. This was similar to the findings of Sudipta et al (2010) ${ }^{1}$. However Mozaffer et $\mathrm{al}(2008)^{2}$ found much higher association of splenomegaly in HL $(81.8 \%)$.

Classifying lymphomas as $\mathrm{B}$ and $\mathrm{T}$-cell type provides significant prognostic information to the oncologists. Recognizing and diagnosing various subtypes of T-cell lymphomas according to WHO 
classification is important as the prognosis of T-cell lymphomas is poorer than B-cell lymphomas. ${ }^{15}$ Among NHL encountered, B-cell type was the most predominant type constituting $71.4 \%$ while T-cell and NK cell type was $28.6 \%$. This was in concordance with other studies by Jin-Man et al $(2011)^{10}$, Sun et al $(2012)^{11}$ and Santosh et al $(2013)^{12}$.

The most common type of lymphoid neoplasms seen all over the world despite considerable geographical variation is the B cell type. The most common histological subtype encountered in our study is DLBCL $(66.7 \%)$ followed by FL $(10 \%)$. This was in comparison with other studies by Wei et al $(2011)^{8}$, JinMan et al $(2010)^{10}$, Sun et al $(2012)^{11}$ and Santosh et al $(2013)^{12}$.

Follicular lymphoma occurs more frequently in India $^{8}$.The molecular pathogenesis of follicular lymphoma in Western countries has been hypothesised to be different from those of Asian follicular lymphoma based on the differences in the frequencies of translocations of bcl-2 in these geographic regions ${ }^{6}$.Naresh et al proposed that, in developing countries, the low rates of FL compared to DLBCL may be due to progression of previously undiagnosed FL. He also postulated that unique regional, genetic or environmental factors may have contributed to such progression. ${ }^{14}$ Other subtypes of lymphomas encountered were LPL (6.7\%), plasma cell myeloma (6.7\%), mantle cell lymphoma(3.3\%), SLL (3.3\%) and ENMZBCL (3.3\%).

Plasma cell neoplasms was seen in $6.7 \%$ of cases, which was also observed by Sun et al (2012) ${ }^{11}$. Although these data effectively characterize the subtype spectrum of lymphomas, most of the other studies have excluded the plasma cell neoplasms. Therefore, these results may not be comparable with population-based incidence data for the entire spectrum of WHO classification. Both REAL and WHO classification encompass not only Hodgkin lymphoma and non-Hodgkin lymphoma but also plasma cell neoplasm and lymphoid leukemia, which represent solid and circulating phases of the same disease.

One case of CLL/SLL was encountered in our study. According to Waravita et al, the prevalence of CLL/SLL remains low in south and east Asian countries highlighting that genetic bias and host related genetic factors play a role in the pathogenesis. ${ }^{6} \mathrm{ENMZBCL}$ in thyroid was seen in one case in the study group. ENMZBCL is thought to have an autoimmune etiology. ${ }^{15}$

Extranodal NK/T cell lymphoma, nasal type (25.1\%) was the most common subtype of T-cell lymphoma encountered, followed by anaplastic large $\mathrm{T}$ cell Lymphoma (16.7\%) and mycosis fungoides (16.7\%). Sun et al also observed extranodal NK/T cell lymphoma, nasal type as the most common histological subtype. ${ }^{11}$ It is observed that extranodal NK/T-cell lymphoma is more prevalent in Asia and is closely related to Epstein Barr virus infection. This suggests an inherited or acquired impaired immunity against EBV, which has a genetic basis. ${ }^{11}$ Other subtypes of T-cell lymphoma observed were angioimmunoblastic $\mathrm{T}$ cell lymphoma (8.3\%), peripheral $\mathrm{T}$ cell lymphoma $(8.3 \%)$, prolymphocytic lymphoma $(8.3 \%)$ and primary cutaneous lymphoma $(8.3 \%)$. There was varied frequency of peripheral $\mathrm{T}$ cell lymphoma in studies like Jin Man et al $(2011)^{10}$ and Wei et al $(2010)^{8}$ compared to our study. This is more likely because of better defined diagnostic criteria for T-lineage lymphomas in the WHO classification (2008) compared with the REAL classification. Also the better classification of a T-lineage lymphoma of unspecified subtype using

NHL has marked variability in morphological features, growth pattern, antigenic phenotype and biological behaviour. Multi-disciplinary approach for the diagnosis of NHL is required which includes routine morphologic evaluation, immunophenotypic characterization and genotypic studies. ${ }^{16}$

Immunohistochemistry was done on all 50 cases of malignant lymphomas in our study. Various antibodies used were of Pan B-cell and Pan T-cell type. Pan B-cell markers were CD 20,19, 79a, 5, 23, 10, 138, BCL 2, BCL-6, cyclin D1, Ki-67 and Pan T-cell markers included CD 3, 5, 7, 8, 30, 43, 56, 99, Tdt, EBV-LMP1, ki-67.

Our study showed $71.4 \%$ of B cell type and $28.6 \%$ of T cell type of NHL. Further subtyping of B and T cell lymphomas were done based on the histopathological features using specific markers.

Grading of NHL was done according to working formulation classification. Dividing the lymphomas into low, intermediate, and high-grade categories helps in the management of the patients and has prognostic implications. In our study, $59.5 \%$ of NHL were classified as intermediate grade, followed by $19 \%$ as low grade and $6.7 \%$ as high grade.

Majority of the intermediate grade lymphomas were of both $\mathrm{B}$ and $\mathrm{T}$ cell types which was in concordance with study by Suhail et al. ${ }^{16}$ Among high grade lymphomas, majority of the cases were of $\mathrm{T}$ cell type $(25 \%)$, while B cell lymphomas $(23.3 \%)$ accounted for higher number of low grade lymphomas.

The most frequent histological subtype of HL in the current study is mixed cellularity $(50.0 \%)$ followed by nodular Sclerosis (37.5\%). This was in concordance with other studies like Yukiko et al (2011), Sun et al (2012) ${ }^{11}$ and Santosh et al $(2013)^{12}$. EBV is more likely to be associated with a mixed cellularity subtype than nodular sclerosis. The increased incidence of mixed cellularity subtype in Asian countries may be due to higher risk of childhood exposure to EBV. ${ }^{12}$ Jin man et al(2011) found a higher incidence of nodular sclerosis type of CHL compared to MCCHL. ${ }^{10}$

Bone marrow study is essential for staging of lymphomas. Bone marrow involvement by the neoplastic process was detected in $23.8 \%$ of cases of NHL and in $12.5 \%$ cases of HL in our study. Santosh et al 
$(2013)^{12}$ and Sudipta et $\mathrm{al}(2010)^{1}$ also observed a higher incidence of involvement of bone marrow in NHL compared to HL.

In our study, bone marrow was involved in subtypes of NHL like DLBCL, LPL, plasma cell myeloma, SLL, AITCL and prolymphocytic T cell lymphoma. MC-CHL showed bone marrow involvement in HL. Other reactive histological changes observed in uninvolved marrow were erythroid hyperplasia, myeloid hyperplasia with increase in eosinophilic precursors, reactive plasmacytosis, and mild megakaryocyte hyperplasia.

The therapeutic strategies are guided by the clinical features and histological findings. The majority of patients are treated in accordance with the staging done by Ann Arbor staging system.

In our study, majority of the patients with NHL were in stage IV $(57.2 \%)$, followed by stage III (26.2\%), II (9.5\%) and I (7.1\%) which was also observed by Mozaffer et al (2008) ${ }^{2}$. However study by Sudipta et al (2010) ${ }^{1}$ had higher incidence of patients presenting in stage III.

In HL, $50 \%$ of the patients in our study were in stage II. Majority of the patients presented in higher stage of the disease in studies done by Mozaffer et al (2008) ${ }^{2}$ and Sudipta et al (2010). ${ }^{1}$ This is probably due to late clinical manifestations, lack of knowledge and awareness about the disease.

\section{Conclusion}

There is increase in the incidence of lymphomas in India and worldwide. Lymphoma is diagnosed based on morphology, a limited panel of immunophenotyping studies, and relevant clinical information. There is a sharp rise in the incidence of extranodal primary lymphoma with wide variations in the presentation of the disease due to involvement of gastrointestinal tract, central nervous system and skin. In non-Hodgkin lymphoma, B-cell subtype is more common than T-cell subtype. Diffuse large B cell lymphoma is the most frequent type of B-cell lymphoma and extranodal NK/T cell lymphoma is the most frequent type of $\mathrm{T}$-cell lymphoma. Mixed cellularity classical Hodgkin lymphoma is the most common type of Hodgkin lymphoma. Bone marrow is more often involved in non-Hodgkin lymphoma than Hodgkin lymphoma. Grading and staging of lymphomas has therapeutic and prognostic implications. A general awareness regarding the clinical manifestations of lymphomas, along with usage of advanced investigative techniques may lead to early precise diagnosis and proper management of this potentially curable disease.

Fig 6a

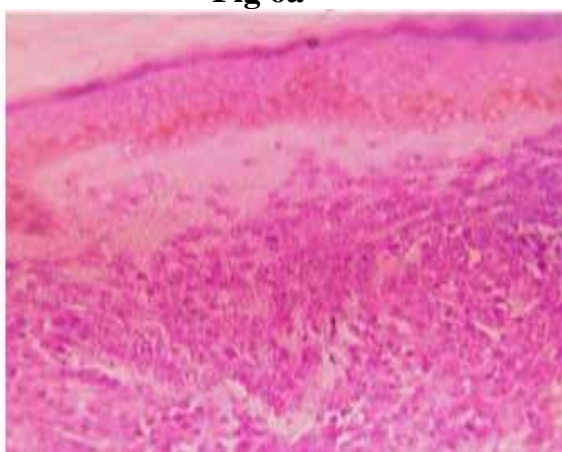

6b

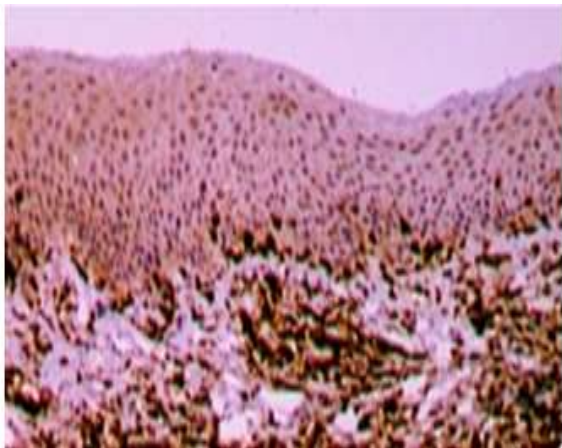

6a.Mycoses fungoides showing grenz zone with infiltration of neoplastic lymphocytes in the upper dermis $(\mathrm{H} \&$ E 100X).

6b. Cytoplasmic membranous CD3 positivity in T-cell lymphoma of skin (Immunoperoxidase stain 40X)

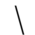

Fig 7a $7 b$
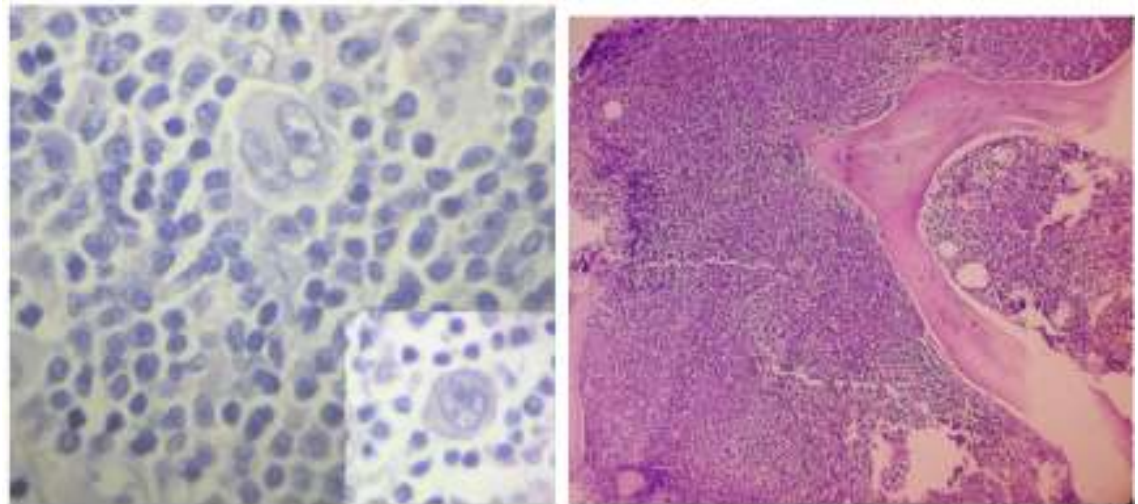

7a. Classic Reed-Sternberg in Hodgkin lymphoma (H \& E 1000X)

7b.Bone marrow biopsy showing diffuse interstitial type infiltration of lymphoma (H \& E 40X) 


\section{References}

[1]. Chakrabarti S, Sarkar S, Goswami BK, Mondal S, Roy A, Das S. Hodgkin and Non-Hodgkin Lymphomas in an Indian Rural Medical Institution: Comparative Clinicopathologic Analysis. Asian Pac J Cancer Prev. 2010;11(6):1605-8.

[2]. Hingorjo MR, Syed S. Presentation, staging and diagnosis of lymphoma: a clinical perspective. J Ayub Med Coll Abbottabad. 2008 Oct-Dec;20(4):100-3.

[3]. Sharma M, Mannan R, Madhukar M, Navani S, Manjari M, Bhasin T S, et al. Immunohistochemical analysis of Non- Hodgkin lymphoma spectrum according to WHO/REAL Classification: A Single Centre Experience from Punjab, India. J ClinDiagn Res. 2014 Jan;8(1):46-9.

[4]. Roy A, Kar R, Basu D, Badhe BA. Spectrum of histopathological diagnosis of lymph node biopsies: a descriptive study from a tertiary care centre in South India over 5 1\%2 years. Indian J PatholMicrobiol. 2013 Apr-Jun;56(2):103-8.

[5]. Yang QP, Zhang WY, Yu JB, Zhao S, Xu H, Wang WY, et al. Subtype distribution of lymphomas in Southwest China: Analysis of 6,382 cases using WHO classification in a single institution. DiagnPathol. 2011; Aug:1-77.

[6]. Waravita TS, Wijetunge TS, Ratnatunga NV. Pattern of lymphoma subtypes in a cohort of Sri Lankan patients. Ceylon Med J. 2015 Mar;60(1): 13-7.

[7]. Sader-Ghorra C, Rassy M, Naderi S, Kourie HR, Kattan J. Type distribution of Lymphomas in Lebonon: Five -year single institution experience. Asian Pac J Cancer Prev. 2014;15(14):5825-8.

[8]. Chen WL, Tsai WC, Chao TY, Sheu LF, Chou JM, Kao WY, et al. The clinicopathological analysis of 303 cases with malignant lymphoma classified according to the World Health Organization classification system in a single institute of Taiwan. Ann Hematol. 2010 Jun;89(6):553-62.

[9]. Aoki R, Karube K, Sugita Y, Nomura Y, Shimizu K, Kimura Y, et al. Distribution of malignant lymphoma in Japan: analysis of 2260 cases, 2001-2006. Pathol Int. 2008 Mar;58:(3):174-82.

[10]. Kim JM, Ko YH, Lee SS, Huh J, Kang CS, Kim CW, et al. WHO Classification of Malignant Lymphomas in Korea: Report of the Third Nationwide Study. Korean J Pathol. 2011;45(3):254-60.

[11]. Sun J, Yang Q, Lu Z, He M, Gao L, Zhu M, et al. Distribution of Lymphoid Neoplasms in China. Analysis of 4,638 Cases According to the World Health Organization Classification. Am J ClinPathol. 2012 Sep;138(3):429-34.

[12]. Mondal SK, Mandal PK, Samanta TK, Chakaborty S, Roy SD, Roy S. Malignant Lymphoma in Eastern India: A retrospective analysis of 455 cases according to World Health Organization classification. Indian J Med PaediatrOncol. 2013 Oct;34(4):242-6.

[13]. Naz E, Mirza T, Aziz S, Danish F, Siddiqui ST, Ali A. Frequency and clinicopathologic correlation of different types of nonHodgkin lymphoma according to WHO classification. J Pak Med Assoc. 2011;61(3):260-3.

[14]. Yaqo RT, Hughson MD, Sulayvani FK, Al-Allawi NA. Malignant lymphoma in Northern Iraq: A retrospective analysis of 270 cases according to the World health Organisation.Indian J Cancer. 2011 Oct-Dec; 48(4):446-51.

[15]. Mushtaq S, Akhtar N, Jamal S, Mamoon N, Khadim T, Sarfaraz T, et al. Malignant Lymphomas in Pakistan according to WHO Classification of lymphoid neoplasms. Asian Pac J Cancer Prev. 2008 Apr-Jun;9(22):229-32.

[16]. Muzaffar S, Pervez S, Aijaz F, Aziz SA, Hasan SH. Immunophenotypic analysis of non-hodgkinLymphoma.J Pak Med Assoc. 1997 Apr;47(4):106-9. 